\title{
Vinculación universitaria: el caso del Centro Soltis para la Investi- gación y la Educación y la comunidad rural de San Juan de Peñas Blancas, Costa Rica
}

\section{University outreach: the case of Soltis Center for Research and Education and the rural community of San Juan de Peñas Blancas, Costa Rica}

\author{
Alberth Humberto Rojas Carranza ${ }^{1}$ \\ Escuela Técnica Agrícola e Industrial. Santa Clara, San Carlos.
}

\author{
Cristian Moreira-Segura ${ }^{2}$ \\ Tecnológico de Costa Rica.
}

\begin{abstract}
Resumen. Este artículo aborda la relación que existe entre el Centro Soltis para la Investigación y la Educación y la comunidad de San Juan de Peñas Blancas, ubicada en el sector Noreste de Costa Rica. Esta relación se analizó a partir de variables de carácter social, ambiental y económico. Se aplicó un cuestionario de preguntas abiertas y cerradas para obtener la percepción de los líderes comunales (miembros de juntas directivas de grupos comunales organizados), sobre la vinculación que existe. Se consultó a todos los integrantes de esas juntas, un total de 51 personas. La mayoría de los resultados obtenidos evidencian una percepción positiva del trabajo entre el Centro y la comunidad. Se logró determinar que más del $50 \%$ ha participado en capacitaciones ofrecidas por el centro, que los vecinos utilizan información científica que tienen disponible en el recinto universitario y que valoran muy positivamente la contribución a la conservación de la flora y la fauna. En resumen, la mayoría considera que han obtenido beneficios directos e indirectos y estiman que la vinculación con el centro podría ser aún mejor. Como resultado de este proceso de investigación se construye y propone un instrumento para futuras evaluaciones de la vinculación entre el Centro Soltis y la comunidad de Peñas Blancas.
\end{abstract}

Palabras clave. Comunidad rural, vinculación académica, centro de investigación.

\begin{abstract}
This article analyzes the relationship between the Soltis Center for Research and Education and the community of San Juan de Peñas Blancas located in the northern part of Costa Rica. The relationship was analized departing from social, environmental and economic variables. A questionnaire was applied to identify the perception of community leaders (members of boards of organized community groups) about the university outreach. All members of these boards, a total of 51 people, were consulted. Most of the results show a positive perception between the activities of Soltis Center and the community. It was determined that more than $50 \%$ of surveyed communal leaders have participated in training offered by the center, the neighbors use scientific information they have available on campus and greatly appreciate the contribution to the conservation of flora and fauna. In short, most participants considered to have obtained direct and indirect benefits and express that the link with the center could be even better. The research proposes a tool for future evaluations of the link between the Soltis Center and the community of Peñas Blancas.
\end{abstract}

Keywords. Rural community, university outreach, research center.

\footnotetext{
${ }^{1}$ Alberth Humberto Rojas Carranza, coordinador del Diplomado en Servicios Turísticos y Actividades Ecoturísticas, Escuela Técnica Agrícola e Industrial. Santa Clara, San Carlos, Costa Rica. Dirección electrónica: arojas7@gmail.com

${ }^{2}$ Cristian Moreira-Segura, profesor de la Escuela de Idiomas y Ciencias Sociales y del Doctorado en Ciencias Naturales para el Desarrollo, Tecnológico de Costa Rica, Sede de San Carlos. Dirección electrónica: cmoreira@tec.ac.cr
} 


\section{Introducción}

Desde una perspectiva social, las expectativas sobre el papel de las instituciones de educación superior se han "modificado a través del tiempo, incorporando otras visiones a los modelos de universidad, entre estos modelos de universidad social y emprendedora, que contemplan el compromiso de la institución con las problemáticas de su entorno y la contribución al desarrollo regional" (Allende, González y Zanfrillo, 2009, p. 41).

La Universidad es una organización que contribuye al bienestar social, a través del desarrollo de sus tareas básicas como la docencia, la investigación y la extensión (Maldonado y Gould, 1994). Tales contribuciones se crean mediante el fenómeno denominado como pertinencia, el cual hace referencia al establecimiento de múltiples relaciones entre la universidad y su entorno (Plata, 2006, p. 80).

El Centro Soltis al estar adscrito a la Universidad Texas A\&M cumple a nivel local diversos aspectos de la pertinencia de dicha universidad. Las diversas actividades de vinculación con que interactúan las organizaciones educativas y las del medio socio productivo, adquieren especial importancia en la transferencia de los avances científicos y tecnológicos al medio en que se asientan (Allende y otros, 2010, p. 4). Resulta importante, también, tomar en cuenta la responsabilidad social universitaria, definida por Ramos (2010) "como la capacidad de la universidad para difundir y poner en práctica un conjunto de principios y valores generales y específicos" (p.101). La interacción de esta institución social con la sociedad en la cual está inserta, se da de diferentes formas y con estructuras diversas, tanto al interior de la universidad como del entorno social.

En el caso de esta institución, uno de los elementos de vinculación que tienen con la comu- nidad de San Juan es el compartir conocimientos con la comunidad circundante. Como bien plantea Plata (2006, p.83), constituyen el eje para el funcionamiento de las universidades y en la actualidad también se les considera como la base de los sistemas de producción. El apoyo de Soltis al desarrollo de la comunidad es muy importante. En este caso, al estar la universidad enclavada en una zona rural, se espera que apoye desde su quehacer el desarrollo local endógeno. Según explica Luna (2014) “el desarrollo endógeno busca la implementación de una estrategia autóctona de desarrollo, que impulse una economía solidaria, orientada a las necesidades colectivas y a los mercados nacionales, impulsando así, la defensa y la valorización del capital natural, cultural y patrimonial" (p.12). Lo anterior, tomando como punto de partida las potencialidades propias, postula que cada región puede y debe buscar nuevas maneras de inserción en un contexto nacional.

En esa misma línea, Espinoza, Andrade, Chávez, \& Zepeda (2012), indican que el "desarrollo local endógeno, de una región o subregión parte del supuesto de que los pobladores deben asumir el rol de participantes activos y deben de ser los generadores de las ideas que, posteriormente se convertirán en los medios de producción comunitaria, así como promotores de las políticas públicas que habrán de sustentar las acciones o prácticas de dicha planeación" (p.3). Es importante, entonces, alinear los intereses de la universidad con los de los pobladores y los grupos sociales que los representan.

El asociacionismo es un elemento impulsor de desarrollo local, pues es esencial, la integración de la sociedad en la toma de decisiones de forma directa e indirecta. López (2004) indica que "se generan redes de información y conocimiento dentro del ámbito territorial, ya que se favorece la distribución de la información al mayor número de personas posible" (p.358). En este sen- 
tido, el apoyo que puedan dar los profesores, investigadores y estudiantes que visitan el Soltis es muy importante, porque en esa comunidad no hay sede de ninguna universidad estatal o privada costarricense.

Una herramienta de gran utilidad para la planificación estructurada del desarrollo endógeno, la constituye el Diagnóstico Rural Participativo (DRP), definido como un conjunto de técnicas y herramientas que permite a las comunidades realizar su propio diagnóstico y de ahí comiencen a auto gestionar su planificación y desarrollo. De esta manera, los participantes pueden compartir experiencias y analizar sus conocimientos, a fin de mejorar sus habilidades de planificación y acción, al promover la autodeterminación de la comunidad, a través de la participación y en el fomento un desarrollo sostenible (Expósito, 2003, p.7); este mismo autor (2003), indica que el DRP "permite obtener información primaria o de "campo" en la comunidad, prestando especial atención a los diversos grupos representativos, hasta llegar a un autodiagnóstico sobre el estado de sus recursos naturales, su situación económica y social y otros aspectos importantes para la comunidad".

Esta investigación indaga, por primera vez desde la creación del centro, sobre elementos propios de su quehacer y el de la comunidad. El identificar estos elementos de coincidencia y divergencia se convierte en el primer paso para la realización de un trabajo conjunto de planificación del desarrollo comunal. La adecuada realización de un DPR para la comunidad de San Juan debe partir entonces de este primer levantamiento de información.

Sobre el Centro Soltis para la Investigación y la Educación

El Centro Soltis para la Investigación y la Educación en Costa Rica es una institución privada de carácter multidisciplinario que fue donada a la Universidad Texas A\&M (http://soltiscentercostarica.tamu.edu), por el señor William "Bill" Soltis, un ex alumno egresado de la carrera de Ingeniería Mecánica en 1955. Bajo la misión institucional de servir como la representación oficial de la universidad Texas A\&M en Costa Rica, el centro busca facilitar el alcance de múltiples metas operativas de la universidad como ente académico, desarrollando diversas actividades y proyectos de investigación, educación y extensión comunitaria en la comunidad de San Juan de San Isidro de Peñas Blancas, sitio en el cual se mantiene operando desde el 18 de junio del 2009 (E. González, comunicación personal, 5 de Agosto de 2014). En función de la utilidad del área de bosque nuboso donde se ubica el Centro Soltis, de su infraestructura física y de los recursos humanos disponibles, el Centro tiene varias áreas de investigación prioritarias y entre estas destacan las siguientes: el monitoreo de especies con migraciones altitudinales, impactos del cambio climático en la biodiversidad y los ecosistemas locales, manejo integrado de cuencas hidrográficas, inventarios de biodiversidad, ecología y biología de la conservación, enfermedades emergentes y su relación con el cambio climático, conservación de especies en peligro de extinción, ecohidrología, conservación de los

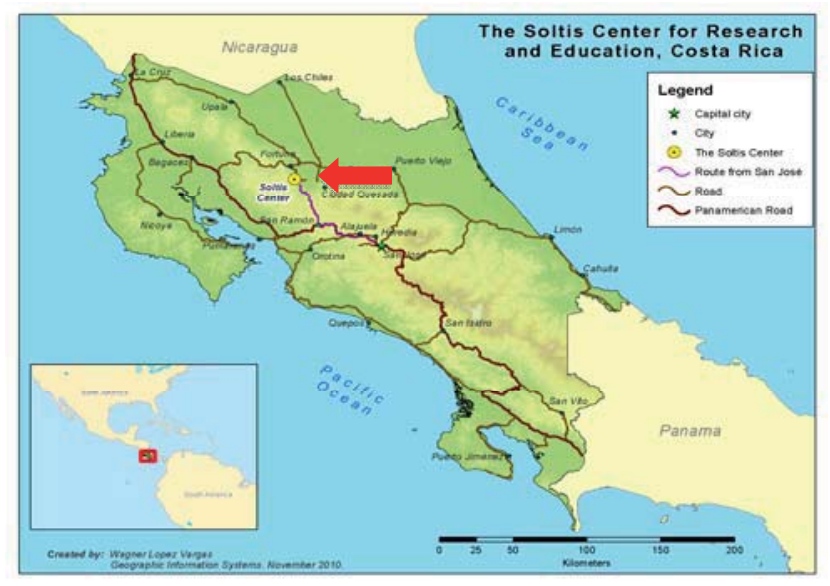

Figura 1. Ubicación del Centro Soltis. Fuente: Centro Soltis, San Juan de Peñas Blancas. 
suelos, agricultura sostenible e hidrometeorología (Houser, Lemmons \& Cahill, 2013).

Desde su establecimiento, el Centro Soltis ha incrementado de forma constante sus operaciones (intercambios académicos, investigación y vinculación, cursos cortos, entre otros) y ha dedicado esfuerzos para incluir en él a la comunidad local y las diversas instituciones presentes en esta. Desarrolla funciones y servicios académicos (cursos, artículos científicos, material audiovisual, entre otros) que han favorecido la producción de nueva información científica, la economía y la conservación de los ecosistemas a nivel local y regional. Se promueve así un desarrollo de tipo sostenible, coordinado y ajustado a las características locales.

\section{Metodología}

Desde el inicio de sus operaciones, el Centro Soltis ha mantenido una relación de cooperación recíproca con la comunidad de San Juan. Sin embargo, hasta la fecha, no se ha desarrollado ninguna investigación que permita conocer los efectos para ambas partes y las áreas en las cuales se presenta una mayor interacción.

Es este vacío de información el que motiva el desarrollo de esta investigación. Debido a eso, se plantea describir las relaciones existentes entre el centro y la comunidad de San Juan como el objetivo terminal de este trabajo.

Dada la diversidad de acciones y áreas temáticas como la educación ambiental, enseñanza de un segundo idioma, turismo científico, por citar algunas, que interactúan conjuntamente al mantener la relación entre el Centro Soltis y la comunidad de San Juan, se da origen a una dinámica y compleja situación de estudio. Por esa razón, para efectos de este trabajo se empleó el enfoque de investigación mixto y descriptivo. Se destaca la atención a los componentes fundamentales de la sostenibilidad, de modo que se tomarán en cuenta los ámbitos social, ambiental y económi$\mathrm{co}$, que tal relación favorece.

\section{Lugar del estudio}

La investigación se realizó en un la Comunidad de San Juan de Peñas Blancas, cantón de San Ramón, provincia de Alajuela, donde se encuentra el Centro Soltis para la Investigación y la Educación. Esta comunidad cuenta con una población que no supera las 700 personas, instaurada en un ambiente rural con una topografía irregular en la que destacan las actividades agropecuarias con un mayor auge del sector agrícola sobre el pecuario. Los orígenes de la comunidad datan de finales de la década de la década de los años 1980 como un proyecto de asentamiento rural para que 24 familias de campesinos locales pudieran tener un terreno propio para cultivar. En sus alrededores, existen exuberantes bosques lluviosos siempre verdes con abundantes fuentes de agua como ríos y quebradas que frecuentemente forman cataratas con una particular belleza escénica. La comunidad limita con una gran franja boscosa, en la que ha sido mínima la investigación científica que se ha desarrollado.

\section{Sobre los participantes del estudio}

Se realizó un censo que incluyó a los 51 miembros que conforman la totalidad de las juntas directivas de los grupos organizados presentes en la comunidad: educativo, económico, agrícola y social. En lo referente a los grupos organizados, estos se dividieron de acuerdo con su ámbito de acción en función de las actividades que realizan. Esto resulta en siete grupos aglomerados de acuerdo con sus características y rango de acción en cuatro ámbitos (educativo, económico, agrícola y social). Para seleccionar a los participantes en el estudio se aplicaron los siguientes criterios: I) Que la persona formara parte de la junta directiva de un grupo organizado de la comunidad. II) Ser mayor de edad. III) Residir en la comunidad donde se localiza el centro de investigación. 
Estos grupos se visualizan con más claridad en la figura 2.

\section{Instrumento utilizado en el estudio}

El instrumento elaborado se aplicó a todos los miembros de las juntas directivas de los grupos organizados de la comunidad de San Juan de San Isidro de Peñas Blancas. Se consideraron tres variables: social, ambiental y económica. Se definieron indicadores que sirvieron de base para la construcción de los ítems del cuestionario aplicado y que constó de 31 ítems: 27 de respuesta cerrada y 4 de respuesta abierta. La primera versión del instrumento fue validada bajo el criterio de expertos con tres profesores-investigadores del Instituto Tecnológico de Costa Rica. Una vez validado por los expertos, se procedió a aplicarlo a 10 miembros de una comunidad que no participó en el estudio, pero que tenía características similares. Esto permitió realizar mejoras al instrumento para la recolección de la información.
El procedimiento metodológico de la investigación constó de tres etapas: inició con el diseño de una matriz con variables, dimensiones e indicadores. Posteriormente, con esta matriz, se construye un instrumento tipo entrevista que contempló las dimensiones e indicadores referentes a las variables planeadas: social, económico y ambiental. Una vez tabulados los resultados de la aplicación del instrumento, se aplica estadística univariada para el análisis de los datos. Más adelante, se realiza una agrupación que permite visualizar los elementos positivos y negativos de la interacción del centro con la comunidad. Finalmente, con estas valoraciones se diseña un instrumento que permite estimar con mayor exactitud el grado de vinculación existente entre el centro y la comunidad. En la tabla 1, Variables de investigación, se detallan las dimensiones y los indicadores que guiaron la construcción del instrumento aplicado.

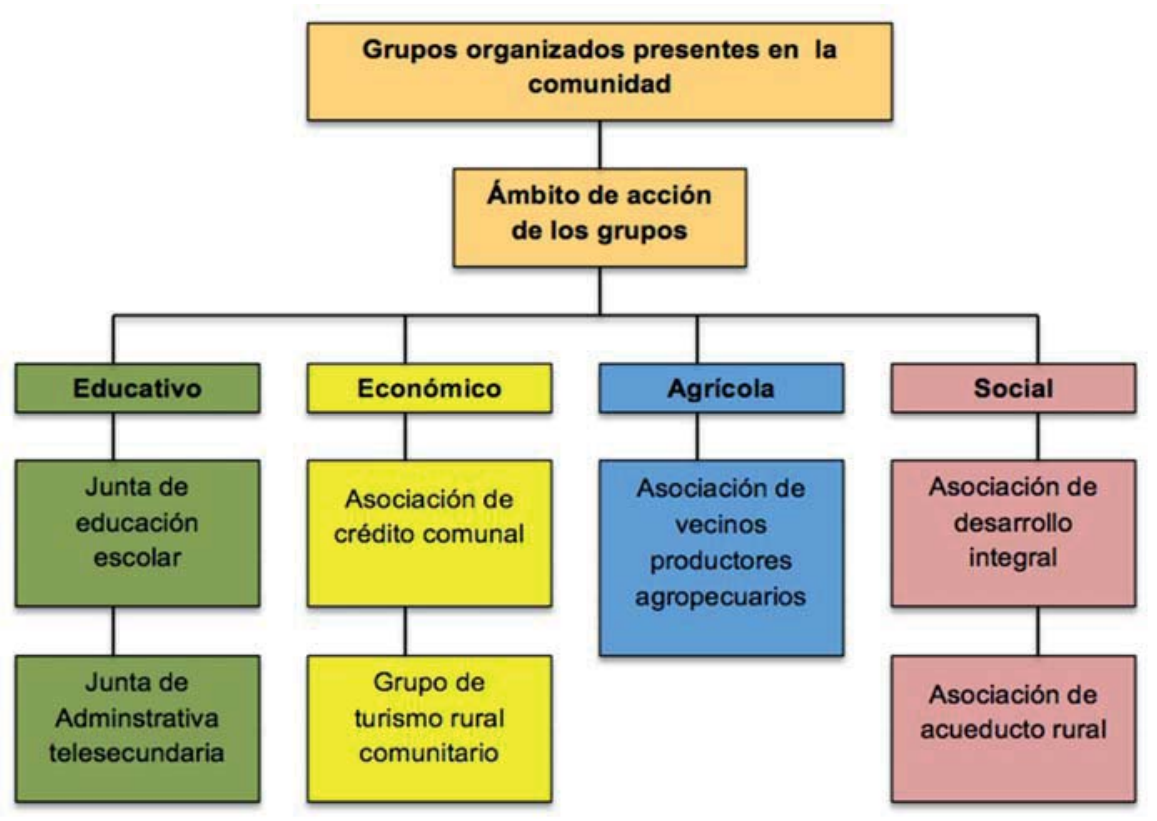

Figura 2. Grupos organizados de la comunidad de San Juan de Peñas Blancas. Nota. Elaboración propia. 


\section{Rojas \& Moreira-Segura}

\section{Tabla 1}

Variables de investigación

\begin{tabular}{|c|c|c|}
\hline Ámbito & Dimensión & Indicadores \\
\hline \multirow{5}{*}{ 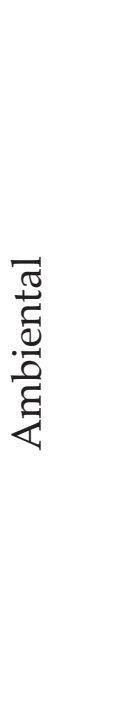 } & Conservación del patrimonio natural. & $\begin{array}{l}\text { - Existencia de áreas dedicadas a la protección } \\
\text { de bosques por el centro o en la comunidad. } \\
\text {-Impacto del centro o contribución de este en la } \\
\text { conservación. }\end{array}$ \\
\hline & Educación e interpretación ambiental. & $\begin{array}{l}\text {-Usos a nivel local de los productos de la inves- } \\
\text { tigación. } \\
\text { - Capacitaciones. }\end{array}$ \\
\hline & $\begin{array}{l}\text { Producción y divulgación de información sobre } \\
\text { el patrimonio natural. }\end{array}$ & $\begin{array}{l}\text {-Artículos, libros publicados, etc. } \\
\text { - Revistas indexas editadas por la institución. }\end{array}$ \\
\hline & Gestión Ambiental & $\begin{array}{l}\text { - Se cuenta con plan de políticas ambientales } \\
\text { (Ahorro eléctrico, agua, manejo de desechos, } \\
\text { plantas de tratamiento, etc.). } \\
\text { - Reutilización y Reciclaje. } \\
\text { - Uso de botaderos legales o ilegales. } \\
\text { - Presentan algún tipo de certificación relaciona- } \\
\text { da con el ambiente }\end{array}$ \\
\hline & Participación en conservación ambiental local & $\begin{array}{l}\text { - Apoyo económico. } \\
\text { - Apoyo organizativo. } \\
\text { - Apoyo científico. }\end{array}$ \\
\hline
\end{tabular}

Apoyo al gobierno local.

-Existen mecanismos formales de colaboración (pasantías, asesorías, actividades con juntas).

- Proyectos asesorados anualmente (ejemplo: educativos, productores locales).

-Proyectos gestionados anualmente.

- Cantidad de grupos que ya han recibido

Apoyo a grupos organizados. apoyo.

- Estímulos a la formación de nuevos grupos organizados.

च્త్ర

- Charlas y talleres temáticos.

- Colaboraciones pedagógicas: giras educativas

- Capacitación a profesores.

- Nivel de satisfacción de los locales con el turis-

Satisfacción de la comunidad. mo científico del centro.

- Cantidad de quejas expresadas por la comunidad sobre el centro.

- Frecuencia de reuniones comunitarias

Participación en actividades comunitarias e índi- • Porcentaje de la población que asiste a las ces de asistencia. reuniones comunitarias. -Índices de Asistencia.

Consumo de productos y servicios locales

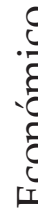

Creación de nuevos empleos

Estímulos a la diversificación económica local.

Apoyo técnico a empresas locales

Inversión en la economía local
- Contratación de mano de obra local. - Compra de insumos a nivel local.

- Cantidad de empleados permanentes.

- Cantidad de empleados temporales.

- Lista de potenciales trabajadores por áreas productivas a nivel local

- Fuentes no tradicionales de empleo.

- Creación de nuevas empleo.

-Asesorías técnicas (producción, comercialización, etc.)

-Percepción de la gente sobre el aporte económico del centro.

Nota. Elaboración propia a partir de Organización Mundial del Turismo (2005) y Aponte (2007). 
Análisis de los datos

Se realizó un análisis univariado a los datos recolectados con el cuestionario que se aplicó a miembros de las juntas directivas de los grupos presentes en la comunidad. Una vez tabulada la información y analizados los datos, se definieron algunos elementos sobresalientes que relacionan al centro con la comunidad. Estos elementos se incluyeron en dos grupos, el que incluye elementos positivos o favorables y el que resume los negativos o desfavorables. Con estos datos se sirvieron de base para la construcción de un instrumento con indicadores, que permitió identificar el grado de vinculación existente entre el Centro Soltis y la comunidad (ver tabla 5).

\section{Resultados y discusión}

A continuación, se presentan los resultados encontrados en el estudio. La tabla 2, incluye la información de los participantes en la investigación. Como se observa, es mayor la participación de mujeres (56\%) en grupos organizados en la comunidad de San Juan de Peñas Blancas que de hombres. En cuanto a las edades, estos grupos comunales están conformados por personas de edad adulta que superan los 42 años de edad (52\%). La mayoría de los participantes está casada (78 \%) y en unión libre (11\%), lo cual apoya un mayor arraigo a la zona y al compromiso de participación, elementos claves para formar parte de un grupo comunal sin fines de lucro. Este

Tabla 2

Características generales de los participantes en el estudio

\begin{tabular}{|c|c|}
\hline Características & Porcentaje \\
\hline Género & 100 \\
\hline Masculino & $44 \%$ \\
\hline Femenino & $56 \%$ \\
\hline Edad & 100 \\
\hline 18 a 25 & $4 \%$ \\
\hline 26 a 33 & $15 \%$ \\
\hline 34 a 41 & $22 \%$ \\
\hline 42 a 49 & $26 \%$ \\
\hline 50 a 57 & $15 \%$ \\
\hline Más de 58 & $18 \%$ \\
\hline Estado civil & 100 \\
\hline Soltero (a) & $7 \%$ \\
\hline Casado (a) & $78 \%$ \\
\hline Viudo (a) & $4 \%$ \\
\hline Unión libre & $11 \%$ \\
\hline Años de residir en la comunidad & 100 \\
\hline Menos de 5 años & $11 \%$ \\
\hline Más de 5 años & $19 \%$ \\
\hline Más de 10 años & $4 \%$ \\
\hline Más de 20 años & $15 \%$ \\
\hline Más de 25 años & $51 \%$ \\
\hline Total de la muestra & 51 \\
\hline
\end{tabular}

Nota: Elaboración propia a partir de datos obtenidos. 
supuesto se comprueba con el resultado que muestra que el $66 \%$ de los participantes tiene más de 20 años de residir en la zona.

Se observa en la tabla 3 que el $93 \%$ de los entrevistados afirma que existen beneficios con la presencia del centro en la comunidad. Además, el resultado es semejante en la percepción que se tiene de que las operaciones que realiza el centro toma en cuenta aspectos relativos a la comunidad en los ámbitos económicos, sociales y ambientales (89 \%). Esta afirmación es comprensible, si tomamos en cuenta que la mayoría de los participantes en el estudio indica que los beneficios que trae el centro son relativamente fáciles de percibir $(67 \%)$, pero el $33 \%$ no puede percibirlos con tanta facilidad. Este resultado es clave porque la comunidad debe de tener mecanismos claros, para identificar el impacto positivo que tiene el centro en la comunidad y sus pobladores y parece que aquí existen, porque el $93 \%$ tiene una imagen positiva del centro como elemento que favorece a la comunidad.
Las manifestaciones de cultura, también, se evalúan de manera positiva con la presencia del centro. Un $93 \%$ afirma que sí tiene un impacto positivo. Es importante agregar que la conformación de grupos organizados se ha favorecido de manera directa o indirecta con la presencia de un centro en la comunidad (78 \%), lo que queda por definir es el cómo y es una pregunta de investigación válida para otro trabajo. De igual manera, se vislumbra a futuro un panorama positivo con la presencia del centro en la zona. La mayoría de los encuestados indican que la vinculación con el Centro Soltis favorecerá las condiciones futuras de la comunidad de San Juan (93\%).

La tabla 4 muestra que la mayoría de los miembros de los grupos organizados (84\%) han tenido acceso a la información sobre capacitaciones realizadas por el centro en favor de la comunidad. Sin embargo, el $16 \%$ no está enterado de esas capacitaciones o no sabe si han existido. Es necesario tener este resultado en cuenta para futuras capacitaciones y así poder incrementar

Tabla 3

Percepción de los pobladores sobre la relación entre el Centro Soltis y la Comunidad de San Juan

\begin{tabular}{lcc}
\hline \multicolumn{1}{c}{ Afirmaciones } & En desacuerdo & De acuerdo \\
\hline $\begin{array}{l}\text { Los grupos organizados se han visto beneficiados con el } \\
\text { establecimiento del Centro Soltis. }\end{array}$ & $7 \%$ & $93 \%$ \\
$\begin{array}{l}\text { Las operaciones del Centro Soltis toman en cuenta aspec- } \\
\text { tos sociales, económicos y ambientales de la comunidad. } \\
\begin{array}{l}\text { Los beneficios que genera el Centro Soltis son fáciles de } \\
\text { percibir y de entender. }\end{array}\end{array}$ & $11 \%$ & $89 \%$ \\
$\begin{array}{l}\text { La promoción y la imagen de la comunidad de San Juan, } \\
\text { mejoraran con la presencia del Centro Soltis. }\end{array}$ & $33 \%$ & $67 \%$ \\
$\begin{array}{l}\text { El Centro Soltis favorece la preservación de las manifesta- } \\
\text { ciones culturales locales. }\end{array}$ & $7 \%$ & $93 \%$ \\
$\begin{array}{l}\text { El Centro Soltis ha favorecido la formación de nuevos } \\
\text { grupos organizados en la comunidad. }\end{array}$ & $22 \%$ & $93 \%$ \\
$\begin{array}{l}\text { La vinculación con el Centro Soltis favorecerá mejores } \\
\text { condiciones futuras para la comunidad de San Juan. }\end{array}$ & $7 \%$ & $78 \%$ \\
\hline
\end{tabular}

Nota: Elaboración propia a partir de datos obtenidos. 
la relación centro-comunidad. En referencia a las capacitaciones que ha realizado el centro con los grupos organizados de la comunidad, la mayoría de los dirigentes comunales (80\%), manifiesta que ha participado en dichas actividades; solo el $14 \%$ no ha participado y el $6 \%$ no responde. Este resultado podría deberse a que existe una buena capacidad de convocatoria por parte del centro o que las capacitaciones son de interés para los grupos organizados. Se puede apreciar que la mayoría ( $80 \%$ ) hace uso de la información científica disponible. Sin embargo, el Centro Soltis debe investigar cuál es el tipo de material o información específica a la que hacen referencia los encuestados para continuar ofreciéndolos y mejorar aún más la vinculación existente.

La vinculación de un centro de investigación científica, en muchas ocasiones, trasciende esa sola idea y se espera que colabore con el desarrollo de la comunidad de forma más amplia. En este sentido, la Tabla 4 muestra la percepción que tienen los miembros de grupos organizados sobre el aporte y el apoyo del centro al comercio local.
La mayoría (80 \%) tiene una apreciación positiva de los aportes que hacen al desarrollo económico de la comunidad. En cuanto a las oportunidades laborales que ofrece el centro a la comunidad, la mayoría (64\%) de participantes indica que sí existen opciones de trabajo, pero el $33 \%$ indica lo contrario. Lo que no queda claro es el medio que se usa para socializar tales oportunidades laborales; esto se debe de retomar en el momento de realizar un plan de vinculación efectivo.

Además de la incorporación de mano de obra local, la utilización de insumos y productos locales son medios para acentuar la vinculación y coadyuvar en la activación de la economía local de la comunidad circundante al Centro Soltis. En este sentido, el Tabla 4 muestra que un porcentaje muy alto de líderes comunales (78 \%) desconoce que el Centro Soltis maneja a nivel interno, listas de proveedores locales para contrataciones de diversos productos y servicios que potencialmente pueden ser provistos localmente antes de tener que contratar o adquirirlos externamente a la comunidad. Este resultado es muy beneficio-

Tabla 4

Percepción sobre las acciones que realiza el centro en la comunidad

\begin{tabular}{lccc}
\hline Acciones realizadas & Sí & No & N/R \\
\hline $\begin{array}{l}\text { Información sobre capacitaciones } \\
\text { organizadas. }\end{array}$ & $84 \%$ & $16 \%$ & - \\
$\begin{array}{l}\text { Participación capacitaciones. } \\
\text { Uso de la información científica. }\end{array}$ & $80 \%$ & $14 \%$ & $6 \%$ \\
$\begin{array}{l}\text { Apoyo al comercio económico } \\
\text { local. }\end{array}$ & $80 \%$ & $6 \%$ & $14 \%$ \\
$\begin{array}{l}\text { Socialización de oportunidades } \\
\text { laborales. }\end{array}$ & $80 \%$ & $20 \%$ & - \\
$\begin{array}{l}\text { Existencia de listas de proveedores } \\
\text { locales. }\end{array}$ & $15 \%$ & $73 \%$ & $7 \%$ \\
$\begin{array}{l}\text { Existencia de aportes al futuro de } \\
\text { la comunidad. }\end{array}$ & $93 \%$ & $7 \%$ & - \\
$\begin{array}{l}\text { Promoción de las manifestaciones } \\
\text { culturales. }\end{array}$ & $80 \%$ & $20 \%$ & - \\
\hline
\end{tabular}


so, porque al estar en esas listas, se le brinda a la comunidad un mayor sentido de pertenencia y de prioridad para ser tomados en cuenta en el desarrollo de las diversas actividades económicas que se realizan.

Muy semejante a los resultados de capacitaciones, o el de uso de la información científica producida, o el de apoyo al comercio local (tabla 4), fueron los de la contribución a la promoción de las manifestaciones culturales de la comunidad, pues la mayoría ( $80 \%$ ) la evaluó como favorable. Este es un hallazgo relevante, pues los esfuerzos del centro no están oficialmente enfocados a este tipo de acciones, puesto que las que lo definen son de un carácter técnico-científico. Estos resultados son útiles porque muchas de las acciones que se realizan se originan de la interacción personal del director del centro y los líderes comunales. Este hecho brinda una posibilidad muy relevante para generar un mayor enlace entre la comunidad y el centro en el ámbito cultural local. De igual manera que en la figura anterior, la tabla 4 muestra que casi la totalidad de los grupos organizados (93\%), reconocen el aporte que hace el centro al futuro de la comunidad. Lo que se requiere es detallar los aspectos puntuales donde se tendrá ese im- pacto esperado con el objeto de fortalecerlos y potenciarlos.

Esto se debe de realizar con un coherente y estrecho trabajo conjunto entre los grupos establecidos en la comunidad y los personeros del Centro Soltis destinados para tal efecto.

La percepción del desempeño del Centro Soltis en conservación de flora y fauna es totalmente, pues el total de los encuestados catalogaron su labor entre excelente y buena. Sin embargo, al analizar individualmente cada categoría, destaca el hecho de que más de la mitad al de los encuestados (52\%) indicó que es buena y dejan abierta la idea de que aún puede ser mejor. Este resultado se vuelve de suma relevancia, puesto que la conservación del patrimonio natural es uno de los elementos claves en los que el Centro Soltis enfatiza sus operaciones. Cabe, aquí, preguntarse si los mecanismos de divulgación que emplea el centro son los adecuados para dar a conocer sus acciones o si más bien lo que se requiere es involucrar más a la comunidad y sus grupos organizados en estos esfuerzos y en las iniciativas que actualmente se desarrollan (ver figura 3).

En cuanto a la labor del centro en conservación en producción y divulgación de información

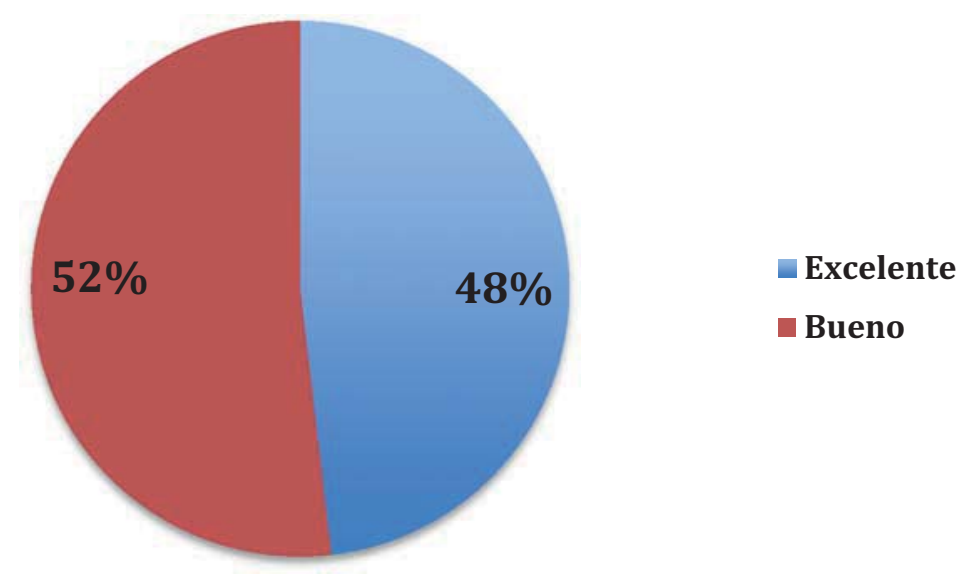

Figura 3. Desempeño del Centro Soltis en conservación de flora y fauna.

Nota. Elaboración propia a partir de datos obtenidos. 


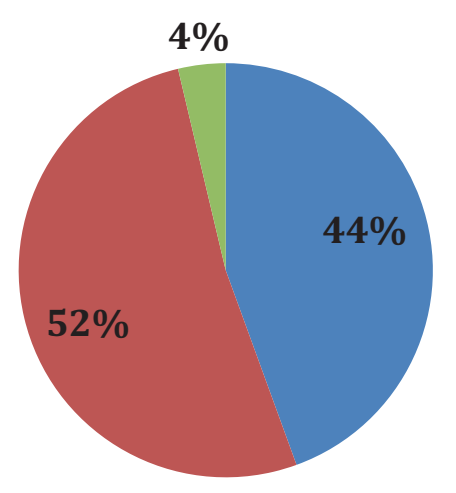

Excelente

Bueno

Malo

Figura 4. Desempeño del Centro en producción y divulgación de información ambiental.

Nota. Elaboración propia a partir de datos obtenidos.

ambiental (ver figura 4), es positiva para la mayoría de los participantes (52\%) y otro número también importante, la considera excelente (44 $\%)$. Cabe mencionar que un número pequeño (4 $\%)$, también indica que esta labor es mala.

La gran mayoría de las personas encuestadas $(95 \%)$ se muestran muy positivas sobre los beneficios futuros (figura 5) que esta vinculación entre el Centro Soltis y la comunidad de San Juan puede generar. Este dato brinda una importante perspectiva que debe ser aprovechada de manera inmediata por el centro para organizar sus lineamientos de vinculación y seleccionar de mejor forma los mecanismos y las actividades que permitan aprovechar este momento de percep- ción positiva para facilitar así cualquier proceso de acercamiento mutuo o inserción de los grupos organizados en dichas medidas.

Una vez hecho el análisis de los resultados obtenidos, se propone un instrumento que toma como base las variables sociales, económicas y ambientales y que puede utilizarlo el centro en estudios futuros para cuantificar el grado de vinculación existente (tabla 5). Para tal efecto, se emplean indicadores y la ponderación de estos con un valor numérico que brinda un rango de valoración sugerido (tabla 6). Este instrumento fue validado en la Comunidad de San Juan y el Centro Soltis y comprobó un grado de vinculación bueno. Su aplicación, arroja un puntaje que

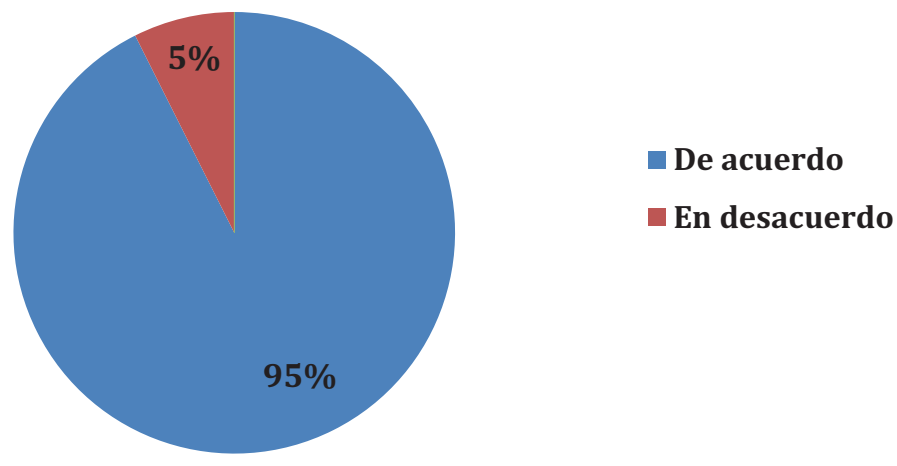

Figura 5. Beneficios futuros de la vinculación del Centro Soltis y la comunidad. Nota. Elaboración propia a partir de datos obtenidos. 
Tabla 5

Instrumento para determinar el grado de vinculación del Centro Soltis con la comunidad

\begin{tabular}{|c|c|c|c|c|c|}
\hline \multirow[b]{2}{*}{ Ámbito } & \multirow[b]{2}{*}{ Indicadores } & \multicolumn{4}{|c|}{ Recurrencia } \\
\hline & & $\begin{array}{l}\text { Actualmente no } \\
\text { se realiza } \\
\text { (0 puntos) }\end{array}$ & $\begin{array}{l}\text { En proceso } \\
\text { de } \\
\text { planeación } \\
\text { (1 puntos) }\end{array}$ & $\begin{array}{l}\text { Se } \\
\text { realiza parcial- } \\
\text { mente } \\
(2 \text { puntos })\end{array}$ & $\begin{array}{l}\text { Se } \\
\text { realiza } \\
\text { regularmente } \\
(3 \text { puntos })\end{array}$ \\
\hline \multirow{12}{*}{$\underset{0}{\tilde{\sigma}}$} & $\begin{array}{l}\text { 1. Cuenta el centro con al menos una línea de acción que obe- } \\
\text { dezca a las inquietudes o necesidades de la comunidad. }\end{array}$ & & & & \\
\hline & $\begin{array}{l}\text { 2. El plan operativo o de trabajo del centro contiene acciones } \\
\text { específicas para vincularse con la comunidad. }\end{array}$ & & & & \\
\hline & $\begin{array}{l}\text { 3. Existen mecanismos claros para canalizar la ayuda que se } \\
\text { puede brindar a la comunidad (formulario, persona de con- } \\
\text { tacto, días de atención a la comunidad, entre otros). }\end{array}$ & & & & \\
\hline & $\begin{array}{l}\text { 4. Los impactos que tienen el centro en la comunidad son } \\
\text { evaluados periódicamente con instrumentos aplicados a los } \\
\text { pobladores aledaños y/o grupos organizados. }\end{array}$ & & & & \\
\hline & $\begin{array}{l}\text { 5. Existen mecanismos claros para dar a conocer a los grupos } \\
\text { organizados los objetivos y el campo de acción del centro. }\end{array}$ & & & & \\
\hline & $\begin{array}{l}\text { 6.El centro ha brindado apoyo técnico grupos organizados de } \\
\text { la comunidad ( hay evidencia: fotografías, listas de asistencia, } \\
\text { cartas de agradecimiento, reconocimientos, entre otros) }\end{array}$ & & & & \\
\hline & $\begin{array}{l}\text { 7. Se ha apoyado a los grupos organizados y la comunidad } \\
\text { con trabajos o investigaciones para resolver problemáticas } \\
\text { locales. }\end{array}$ & & & & \\
\hline & $\begin{array}{l}\text { 8. Los grupos culturales locales tiene representación de fun- } \\
\text { cionarios del centro. }\end{array}$ & & & & \\
\hline & $\begin{array}{l}\text { 9. Las actividades de carácter científico que organiza el centro } \\
\text { son de interés para la comunidad. }\end{array}$ & & & & \\
\hline & $\begin{array}{l}\text { 10.Las iniciativas educativas a nivel local y regional son apo- } \\
\text { yadas por el centro y sus funcionarios (facilita instalaciones, } \\
\text { tutores, equipo, reactivos, especies animales y vegetales, entre } \\
\text { otros) }\end{array}$ & & & & \\
\hline & $\begin{array}{l}\text { 11. Al existir problemas locales, el centro tiene una participa- } \\
\text { ción activa en su resolución. }\end{array}$ & & & & \\
\hline & $\begin{array}{l}\text { 12. La comunicación con la comunidad y sus grupos es fluida } \\
\text { (existen evidencias de solicitudes, formularios de solicitud, } \\
\text { correos-buzón de sugerencias, libro de visitas, etc.) }\end{array}$ & & & & \\
\hline \multirow{3}{*}{ 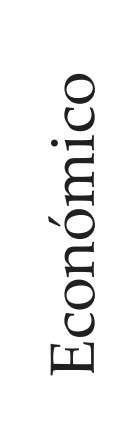 } & $\begin{array}{l}\text { 13. El centro cuenta con una política institucional sobre la } \\
\text { anuencia para el acceso de la comunidad a recursos educati- } \\
\text { vos como internet, contactos profesionales o especializados, } \\
\text { materiales didácticos de bibliotecas, espacio físico de instala- } \\
\text { ciones para reuniones. }\end{array}$ & & & & \\
\hline & $\begin{array}{l}\text { 14. Existe un programa de transferencia de conocimientos o } \\
\text { capacitaciones técnicas del centro hacia la comunidad y los } \\
\text { grupos organizados. }\end{array}$ & & & & \\
\hline & $\begin{array}{l}\text { 15. El centro dispone de los mecanismos para dar a conocer } \\
\text { a la comunidad aledaña cuando requiere contratar personal } \\
\text { temporal o permanente antes que a la comunidad nacional. }\end{array}$ & & & & \\
\hline
\end{tabular}

Continúa... 


\section{Vinculación universitaria: el caso del Centro Soltis}

Ámbito

Indicadores

Recurrencia

\begin{tabular}{|c|c|c|c|}
\hline \multicolumn{4}{|c|}{ Recurrencia } \\
\hline $\begin{array}{l}\text { Actualmente no } \\
\text { se realiza } \\
\text { (0 puntos) }\end{array}$ & $\begin{array}{l}\text { En proceso } \\
\text { de } \\
\text { planeación } \\
\text { (1 puntos) }\end{array}$ & $\begin{array}{l}\text { Se } \\
\text { realiza parcial- } \\
\text { mente } \\
\text { (2 puntos) }\end{array}$ & $\begin{array}{l}\text { Se } \\
\text { realiza } \\
\text { regularmente } \\
\text { (3 puntos) }\end{array}$ \\
\hline & & & \\
\hline & & & \\
\hline & & & \\
\hline & & & \\
\hline & & & \\
\hline & & & \\
\hline & & & \\
\hline & & & \\
\hline & & & \\
\hline & & & \\
\hline & & & \\
\hline & & & \\
\hline
\end{tabular}

Nota. Elaboración propia a partir de los resultados de la investigación.

Tabla 6

Clave general de interpretación para determinar el grado de vinculación del centro de investigación-comunidad

\begin{tabular}{|c|c|c|}
\hline Puntaje Obtenido & Rango/Nivel & Observaciones \\
\hline $0-25$ & Malo & $\begin{array}{l}\text { No se visualizan acciones concretas y claras que sugieran una clara intención } \\
\text { de relacionarse más con la comunidad circundante. Se sugiere realizar acciones } \\
\text { correctivas a la mayor brevedad, con el fin de estrechar los lazos entre el centro } \\
\text { y la comunidad aledaña, para esto se recomienda tomar como punto departida } \\
\text { los grupos organizados ya establecidos y definidos. }\end{array}$ \\
\hline $26-49$ & Deficiente & $\begin{array}{l}\text { Existen algunos indicios de que se está en un punto en el que requiere mayor } \\
\text { relación con la comunidad y sus grupos representantes. Es imperativo que se } \\
\text { establezcan acciones claras y concretas para lograr esa vinculación con la comu- } \\
\text { nidad. }\end{array}$ \\
\hline $50-75$ & Bueno & $\begin{array}{l}\text { El puntaje obtenido sugiere que se encuentra en un punto donde se toma en } \\
\text { cuenta la opinión y los intereses de la comunidad aledaña y los grupos organi- } \\
\text { zados. El grado de relación entre la comunidad y el centro es alto, lo cual revela } \\
\text { un alto nivel de vinculación. }\end{array}$ \\
\hline
\end{tabular}

Nota. Elaboración propia a partir del trabajo de campo. 
oscila entre los 50 y los 75 puntos según la descripción sugerida en el tabla 6.

\section{Conclusiones}

-Los resultados obtenidos evidencian la existencia de un grado de vinculación bueno entre el Centro Soltis y la comunidad de San Juan de Peñas Blancas. Esto constituye la base para el establecimiento de una mejor relación futura, que permita incrementar los beneficios de ambas partes a largo plazo.

-Las operaciones del Centro Soltis, en relación con la comunidad de San Juan, toman en cuenta los ámbitos social, ambiental y económico. Se favorece con ello el desarrollo sostenible a nivel local.

- La vinculación representa la acción operativa que más beneficios mutuos genera entre el Centro Soltis y la comunidad local y se refleja en la percepción positiva que tienen los participantes en relación con los beneficios que se tienen con la presencia del centro en la comunidad. Dentro de estos, sobresalen el realce de la imagen de la comunidad, la creación de nuevos grupos organizados y la conservación de las manifestaciones culturales locales.

- Un porcentaje alto de los líderes comunales participantes considera que la relación existente con el Centro Soltis tiene potencial para generar mejores condiciones y reconocen el aporte que hace el centro a los propios grupos a los que pertenecen y al desarrollo futuro de la comunidad.

- Tanto el centro como la comunidad están anuentes a participar de manera más activa en las actividades que se realizan y benefician a la comunidad y que hasta ahora realizan de manera incipiente cada uno de ellos.

-Aunque existe vinculación entre el centro y la comunidad, se requiere realizar un estudio más exhaustivo para determinar con detalle el impacto que se tiene en el desarrollo de la comunidad de San Juan de Peñas Blancas.

-El instrumento creado para identificar el grado de vinculación entre el centro y la comunidad constituye un insumo de gran relevancia para generar la información base para establecer estrategias y acciones específicas para fortalecer y mejorar la relación entre ambos.

\section{Referencias}

Allende, O., González C, María I. y Zanfrillo, A. (2009). Estrategias de vinculación para la transferencia científica y tecnológica al sector productivo. Colóquio Internacional sobre Gestão Universitária na América do Sul, 9, 2527. Diciembre 2009, Florianópolis.

Aponte, C. (2007). Propuesta de indicadores de evaluación de la función de proyección sociall extensión universitaria/interacción en la educación superior. Documento de trabajo ASCU N, Bogotá, Colombia.

Espinoza, R., Andrade, E., Chávez, R. M., \& Zepeda, A. (2012). Desarrollo local endógeno y productos turísticos. Caso Subregión Occidental de Jalisco, México. TURyDES, Revista de Investigación y desarrollo local, 5(13).

Expósito, M. (2003). Diagnóstico rural participativo. Una guía práctica. Santo Domingo: Centro Cultural Poveda. 118 p.

Houser, C., Lemmons, K., \& Cahill, A. (2013). Role of the faculty mentor in an undergraduate research experience. Journal of Geoscience Education, 61(3), 297-305.

Houser, C., Cahill, A., \& Lemmons, K. (2014). Assessment of student and faculty mentor perceptions of an international undergraduate research program in physical geography. Journal of Geography in Higher Education, 38(4), 582-594. 
López , F. (2004). El turismo como factor de desarrollo endógeno: análisis de la asociación EUROEUME.

Luna, S. (2014). Naturaleza, cultura y desarrollo endógeno: Un nuevo paradigma del turismo sustentable. Una experiencia con el grupo étnico Teenek en la Huasteca potosina, México. Fundación Universitaria Andaluza Inca Garcilaso para eumed.net. $354 \mathrm{p}$.

Maldonado, L. y Gould, G.(1994). La vinculación como estrategia de desarrollo en las Universidades Públicas. Publicación trimestral de la asociación nacional de universidades e instituciones de educación superior, XXIII(3), 91, Julio-Septiembre.

Organización Mundial del Turismo (2005). Indicadores de desarrollo sostenible para los destinos turísticos: guía práctica. Madrid: OMT.

Plata, L. (2006). La vinculación Universidad-Sociedad desde una perspectiva social. Educación y Educadores, 9, 2, 79-93.

Ramos, C. (2010). Hacia una cultura de responsabilidad social universitaria. CICAG, 7 (2), 97-113. 